\title{
LP-based Approximation Algorithms for Facility Location in Buy-at-Bulk Network Design ${ }^{\star}$
}

\author{
Zachary Friggstad • Mohsen Rezapour • \\ Mohammad R. Salavatipour • Jose A. Soto
}

Received: date / Accepted: date

\begin{abstract}
We study problems that integrate buy-at-bulk network design into the classical (connected) facility location problem. In such problems, we need to open facilities, build a routing network, and route every client demand to an open facility. Furthermore, capacities of the edges can be purchased in discrete units from $K$ different cable types with costs that satisfy economies of scale.

We extend the linear programming framework of Talwar [IPCO 2002] for the single-source buy-at-bulk problem to these variants and prove integrality gap upper bounds for both facility location and connected facility location buy-at-bulk problems. For the unconnected variant we prove an integrality gap bound of $O(K)$, and for the connected version, we get the first LP-based bound of $O(1)$.
\end{abstract}

Keywords Buy-at-Bulk Network Design · Facility Location · Approximation Algorithm · LP Rounding

\section{Introduction}

We study problems that integrate buy-at-bulk network design into the classical (connected) facility location problem. We are interested in applications with trade-

* A preliminary version of this paper appeared in the Proceedings of the 14th International Symposium on Algorithms and Data Structures (WADS) [4].

Zachary Friggstad

Department of Computing Science, University of Alberta, Edmonton, Alberta, Canada. Email: zacharyf@ualberta.ca. Supported by NSERC and funding from the Canada Research Chairs program.

Mohsen Rezapour

Department of Computing Science, University of Alberta, Edmonton, Alberta, Canada. Email: rezapour@ualberta.ca.

Mohammad R. Salavatipour

Department of Computing Science, University of Alberta, Edmonton, Alberta, Canada. Email: mrs@ualberta.ca. Supported by NSERC.

Jose A. Soto

DIM and CMM, Universidad de Chile, E-mail: jsoto@dim.uchile.cl. Partially supported by FONDECYT grant 11130266 (Chile) and by Núcleo Milenio Información y Coordinación en Redes ICM/FI P10-024F. 


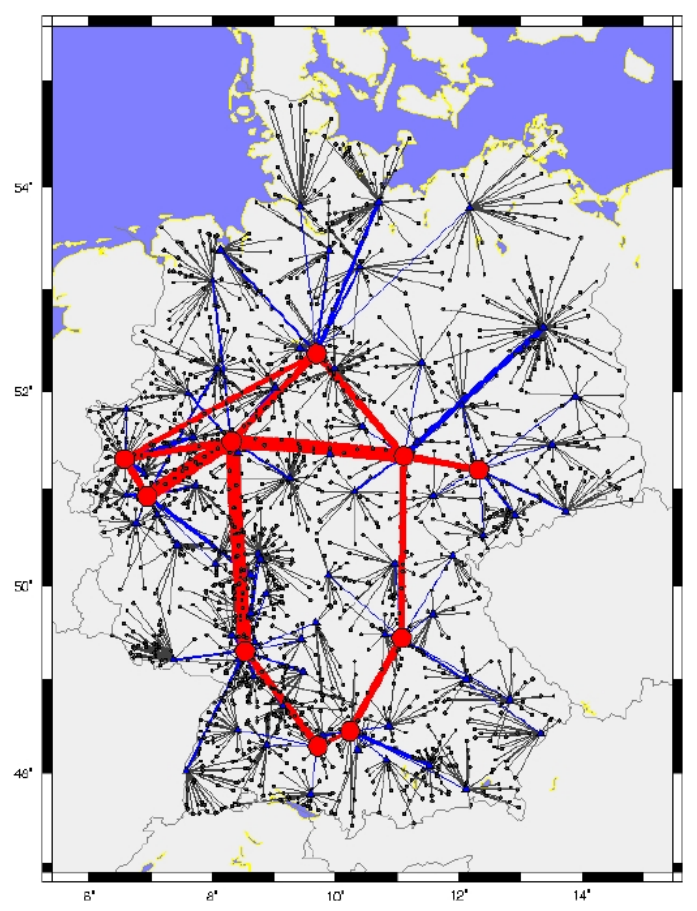

Fig. 1: An example of the fiber optic network, where red lines represent the (national) core network and blue lines represent the (local) access networks.

offs between facility opening and network design costs. Problems of this type arise in the planning of optical access networks in telecommunications, for example. An operator must decide on which nodes to install routing and switching devices (these are called central offices, and represented by facilities) and on which edges to install transmission technologies (represented by so-called cable types) to route traffic demands. In these networks, the traffic originating from each client is sent via tree-like access networks, to its respective facility. A combination of different cable types may be installed on the edges of these access trees to support the traffic flow. This allows for multiple fibers emanating from different clients to share a single, larger cable and the same trunk on their common path towards their common central office. The facilities are connected amongst each other or to some higher network level via a core network of (almost) unlimited capacity, which is required to route the traffic further towards its destination; e.g., see Figure 1.

Designing such a network involves selecting the facilities, connecting them via high-bandwidth links, and dimensioning the access links that are used to route the traffic from the clients to facilities. This can be modeled as a connected facility location with buy-at-bulk edge costs problem, denoted by BBCFL. Formally, we are given a complete graph $G=(V, E)$ with nonnegative edge lengths $c_{e} \in \mathbb{Z}_{\geq 0}$, $e \in E$ satisfying triangle inequality; a set $F \subseteq V$ of facilities with opening costs $\mu_{i} \in \mathbb{Z}_{\geq 0}, i \in F$; and a set of clients $D \subseteq V$ with demands $d_{j} \in \mathbb{Z}_{>0}, j \in D$. We are also given $K$ types of access cables that may be used to connect clients to 


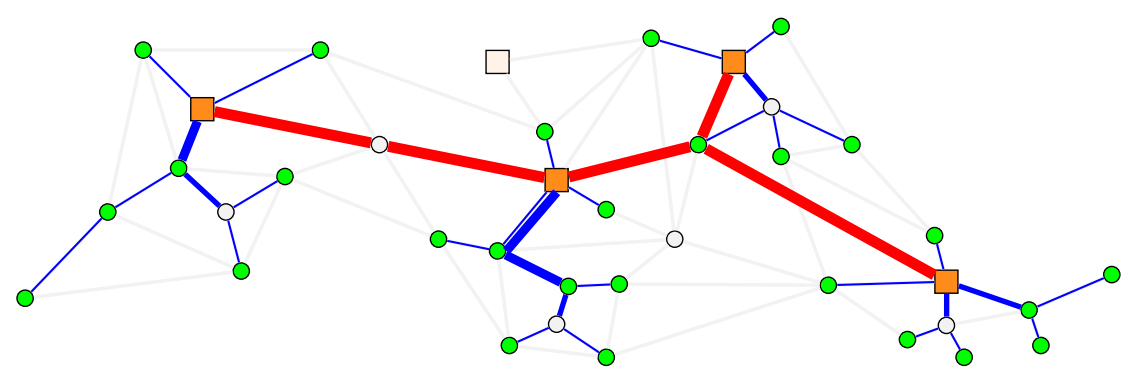

Fig. 2: A feasible solution for BBCFL, where square nodes (in orange) represent (open) facilities, circle nodes in green represent clients, red lines represent core cables, and blue lines (of different thicknesses) represent access cables (of different capacities).

open facilities. A cable of type $i$ has capacity $u_{i} \in \mathbb{Z}_{>0}$ and cost (per unit length) $\sigma_{i} \in \mathbb{Z}_{\geq 0}$. Furthermore, we are given an extra type of cable, called core cable, having a cost (per unit length) of $M>\sigma_{K}$ and infinite capacity, which may be used to connect the open facilities with each other. We assume that access cable types obey economies of scale. That is, $\sigma_{1}<\sigma_{2}<\cdots<\sigma_{K}$ and $\frac{\sigma_{1}}{u_{1}}>\frac{\sigma_{2}}{u_{2}}>\cdots>\frac{\sigma_{K}}{u_{K}}$. A feasible solution (see Figure 2) for BBCFL consists of (1) A subset $F_{0} \subseteq F$ of facilities to open; (2) a Steiner tree of $G$ (core network) connecting all open facilities via core cables; and (3) a forest (access network) connecting all clients to the open facilities. Furthermore, on each edge of this forest we have to specify a list of possibly multiple copies and types of access cables to install, in such a way that the entire demand of each client can be routed along a single path to an open facility. Note that we allow the demand crossing a single edge to use different access cables, but the collection of edges trasversed must be a path in $G$. The objective of BBCFL is to minimize the total cost of opening facilities, and constructing core and access networks; where the cost for using edge $e$ in the core network is $M c_{e}$, and the cost for installing a single copy of access cable of type $i$ on an edge $e$ is $\sigma_{i} c_{e}$.

It is worth noting that we are allowed to install core cables on edges incident to closed facilities, to clients, or even to nodes in $V \backslash(F \cup D)$. Nevertheless, the demand from a client to its facility is not allowed to use core cables. The rationality for this constraint is that in real-life situations core and access networks are run independently. The only way to access from the access network to the core network is via an open facility.

There are various interesting variants of BBCFL that differ with respect to the structure of the access or core network. For example, the planning of water and energy supply networks occur in settings where the consideration of different connection types on the edges of the access network is not motivated by the different capacities but by the different per unit shipping cost of alternative technologies or operational modes. This naturally leads to another interesting variant of the BBCFL problem called connected facility location with deep-discount edge costs problem, denoted by DDCFL. In this problem, instead of capacitated access cables, we are given $K$ discount cable types, where cable type $i$ has a fixed cost (setup cost) of $\sigma_{i}$, a flow dependent incremental cost of $\delta_{i}$, and unbounded capacity. We 


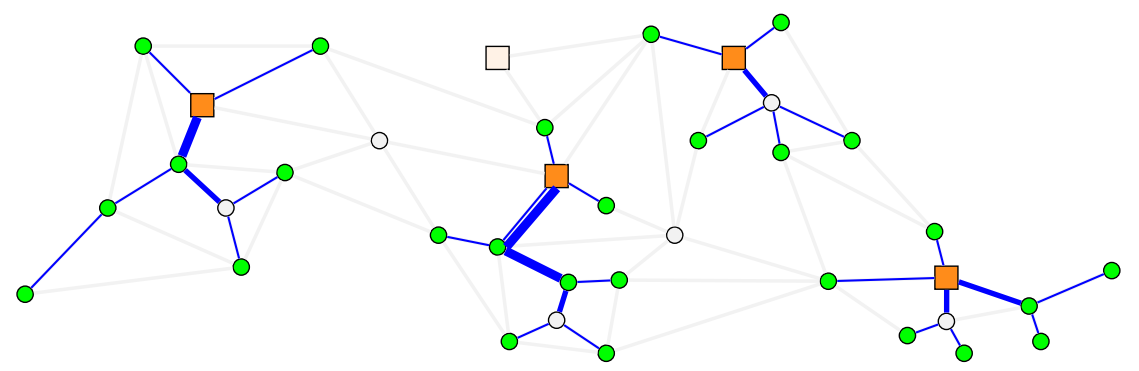

Fig. 3: A feasible solution for BBFL.

assume that $\delta_{1}>\delta_{2}>\cdots>\delta_{k}$ (i.e discount cables obey economies of scale). The cost for installing one copy of discount type $i$ on edge $e$ and transporting $R$ flow units on $e$ is $\left(\sigma_{i}+R \delta_{i}\right) c_{e}$. Yet another interesting variant of BBCFL occurs in logistic networks where the connectivity among facilities is not required, see [14] for more details. This is called facility location with buy-at-bulk edge costs problem, denoted by BBFL; see Figure 3. As with BBCFL, we also consider the deep-discount variant of BBFL, denoted by DDFL, in which we replace the capacitated access cables by discount cable types.

Previous Work

The BBFL problem was first considered by [13]. They show that BBFL can be seen as a special case of the Cost-Distance problem, and thereby provide the first randomized approximation algorithm with approximation guarantee $O(\log (|D|))$ for this problem. Their algorithm works for the more general version of single-sink non-uniform buy-at-bulk network design where one has a different set of cable types for each edge. The algorithm of [13] was then derandomized by [2] who show that the integrality gap of the Cost-Distance problem is $O(\log (|D|))$. To the best of our knowledge, this is the only LP-based approximation algorithm that works for BBFL. Later, an $O(K)$ approximation for BBFL was developed by [14] (where $K$ is the number of cable types), who extended the combinatorial approximation algorithm of [9] that was devised for the single-sink (uniform) buy-at-bulk network design problem.

The BBCFL problem was recently considered by Bley and Rezapour [1] who designed an approximation algorithm based on the random sampling techniques, achieving a 192-approximation for BBCFL. They also obtained a 384approximation to DDCFL via a simple factor 2 reduction between DDCFL and BBCFL. We note that one can show that an $\rho$-approximation to BBCFL gives a $(1+\epsilon) \rho$-approximation to DDCFL; see Lemma 2.2 in [8] for more details. This together with the result of Bley and Rezapour [1] yields a $(192+\epsilon)$-approximation to DDCFL.

The unsplittable Single-Sink (uniform) Buy-at-Bulk network design problem (uSSBB), also known as the single sink problem, can be seen as a further simplification of BBCFL in which the set of interconnected open facilities are given in advance. Several approximation algorithms for uSSBB have been proposed in 
the literature. Using LP rounding techniques, Garg et al. [5] developed an $O(K)$ approximation. The first constant factor approximation for this problem is due to Guha et al. [9]. Talwar [17] showed that an LP formulation of this problem has a constant integrality gap and provided a 216 approximation. Using sampling techniques, this factor was reduced to 145.6 by [11], and later to 40.82 by [6].

The Connected facility location problem (ConFL) is the special case of BBCFL with only one access cable type of unit capacity. Gupta et al. [10] obtained a 10.66approximation for this problem, based on LP rounding. Swamy and Kumar [16] improved the approximation ratio to 8.55, using a primal-dual algorithm. Using sampling techniques, the guarantee was later reduced to 4 by [3], and to 3.19 by $[7]$.

\section{Our Results}

The focus of our work in this paper is on LP-based techniques. We extend the LP-based approximation for uSSBB by [17] to both buy-at-bulk facility location and buy-at-bulk connected facility location problems and prove integrality gap upper bounds for these problems.

Similar to previous work, one can show that a $\rho$-approximation algorithm for DDCFL gives a $2 \rho$-approximation algorithm for BBCFL. For going in the other direction, however, there is only a factor of $(1+\epsilon)$ lost: $\rho$-approximation to BBCFL gives a $(1+\epsilon) \rho$-approximation to DDCFL; see [8] for more details.

Since the integrality gap of the natural flow-based formulation for BBCFL can be arbitrarily large, we focus on the DDCFL problem. In Section 2, we present a strong flow-based IP, namely (IP-DDCFL), model for DDCFL. Our main result is the following.

Theorem 1 The integrality gap of (IP-DDCFL) is at most 234.

Thus, we obtain the first LP based (deterministic) algorithm for DDCFL and thereby for BBCFL.

Using similar techniques, we finally obtain a new LP-based approximation algorithm for the buy-at-bulk facility location problem in Section 3. We propose a flow-based IP, namely (IP-DDFL), formulation for DDFL and obtain the following result.

Theorem 2 The integrality gap of (IP-DDFL) is at most $O(K)$.

This matches the approximation guarantee of the combinatorial algorithm of [14], improving the LP-based $\mathrm{O}(\log (|D|))$-approximation obtained by [2]

The reason why we get a better guarantee for BBCFL, even though it may seem more difficult than BBFL, is that the extra constraints in (IP-DDCFL) that ensure connectivity among open facilities are helpful in bounding the integrality gap.

\section{Buy-at-Bulk Connected Facility Location}

Recall that the only difference between BBCFL and DDCFL is due to the access cable models considered for each variant: In BBCFL, an access cable of type 
$k$ has a fixed capacity $u_{k} \in \mathbb{Z}_{>0}$ and fixed setup cost $\sigma_{k} \in \mathbb{Z}_{\geq 0}$; whereas, in DDCFL, an access cable of type $k$ has a setup cost $\sigma_{k} \in \mathbb{Z}_{\geq 0}$, a flow dependent cost of $\delta_{k} \in \mathbb{Z}_{>0}$, and unbounded capacity. As has been observed in the earlier works, one can transform between buy-at-bulk and deep-discount variants of the problem with factor 2 loss: Given an instance of BBCFL, one can consider a corresponding DDCFL instance by omitting the cable capacity $u_{k}$ of each access cable $k$ and setting its flow dependent cost to $\delta_{k}:=\frac{\sigma_{k}}{u_{k}}$. It is not hard to see that $\left\lceil\frac{D_{e}}{u_{k}}\right] \sigma_{k} c_{e} \leq\left(\sigma_{k}+D_{e} \frac{\sigma_{k}}{u_{k}}\right) c_{e} \leq 2\left\lceil\frac{D_{e}}{u_{k}}\right\rceil \sigma_{k} c_{e}$ holds for any edge $e$, where $D_{e}$ is the total demand carried by edge $e$. Hence the total cost of the access cable installation of any solution of BBCFL is always within a factor of two of the cost of the same access cable installation as the solution to the corresponding modified instance of DDCFL, implying that an $\rho$-approximation to DDCFL gives a $2 \rho$-approximation to BBCFL.

It is not hard to show that the natural flow-based integer linear program for BBCFL has unbounded integrality gap, using the fact that an IP formulation of BBCFL has to purchase capacities in discrete unites to support the demand carried by each edge, while the LP relaxation can pay much less for supporting that demand by only using the last cable type, with the lowest cost per capacity ratio, fractionally.

Hence in this section we focus on the DDCFL problem and get an Integrality gap of $O(1)$ for the underlying LP of the problem, thereby obtaining a new LPbased $O(1)$-approximation algorithm for the BBCFL problem.

\subsection{IP Modeling of DDCFL}

We write a flow-based IP formulation for DDCFL. We assume w.l.o.g. that a particular facility $r$ is open and thus it belongs to the core network in the optimal solution and that $D \cap F=\emptyset$. Also, to simplify the description of our algorithm it will be useful to add an artificial root client $r^{*}$ with unit demand, connected to $r$ by an edge of 0 length. For each edge we create a pair of anti-parallel directed arcs, with same length as the original one. Let $\mathbf{E}$ be the set of these arcs. The undirected version of an $\operatorname{arc} e \in \mathbf{E}$ is denoted by $\bar{e}$.

For every $e \in \mathbf{E}$, cable type $k \in[K]=\{1, \ldots, K\}$ and client $j \in D$, the variable $f_{e ; k}^{j}$ indicates if flow from client $j$ uses cable type $k$ on arc $e$; for $\bar{e} \in E$ and $k \in[K]$, $x_{\bar{e}}^{k}$ indicates if cable type $k$ is installed on edge $\bar{e} ; z_{\bar{e}}$ indicates if the core cable is installed on edge $\bar{e}$; and $y_{i}$ indicates if facility $i$ is opened.

The opening cost $C^{\mathrm{op}}$, the core cost $C^{\mathrm{core}}$, the fixed cost $C^{\text {fixed }}$ and the routing cost $C^{\text {route }}$ of a solution are defined as

$$
\begin{aligned}
& C^{\text {op }}=\sum_{i \in F} \mu_{i} y_{i} ; \quad C^{\text {core }}=M \sum_{\bar{e} \in E} c_{\bar{e}} z_{\bar{e}} ; \quad C^{\text {fixed }}=\sum_{k=1}^{K} C_{k}^{\text {fixed }} ; \quad C^{\text {route }}=\sum_{k=1}^{K} C_{k}^{\text {route }}, \\
& \text { where } C_{k}^{\text {fixed }}=\sigma_{k} \sum_{\bar{e} \in E} c_{\bar{e}} x_{\bar{e}}^{k}, \quad \text { and } \quad C_{k}^{\text {route }}=\delta_{k} \sum_{j \in D} d_{j} \sum_{e \in \mathbf{E}} c_{\bar{e}} f_{e ; k}^{j},
\end{aligned}
$$

represent the fixed cost and routing cost of the cables of type $k$, respectively.

We use the notation $\delta^{+}(S)=\{(u, v) \in \mathbf{E}: u \in S, v \notin S\}, \delta^{-}(S)=\delta^{+}(V \backslash S)$, $\delta(S)=\{u v \in E: u \in S, v \notin S\}$ for each $S \subseteq V$ and $\delta^{+}(v)=\delta^{+}(\{v\})$ for each 
$v \in V$. Given a set of cables $I \subseteq[K]$ and a client $j \in D$, we define the access flow on $e \in \mathbf{E}$ with respect to $I$ and $j$ as $f_{e ; I}^{j}=\sum_{k \in I} f_{e ; k}^{j}$; and the net in-flow on a vertex $v \in V$ with respect to $I$ and $j$, as $g_{I}^{j}(v)=\sum_{e \in \delta^{-}(v)} f_{e ; I}^{j}-\sum_{e \in \delta^{+}(v)} f_{e ; I^{j}}^{j}$. We also define $h_{i}^{j}=\max \left\{g_{[K]}^{j}(i), 0\right\}$ for $j \in D$ and $i \in F$. Formally, this quantity indicates whether facility $i$ is serving client $j$.

With all the notation above, our integer program formulation is as follows.

$$
\begin{array}{rlrl}
\min C^{\text {op }}+C^{\text {core }}+C^{\text {fixed }}+C^{\text {route }} & & \text { (IP-DD } \\
g_{[K]}^{j}(j) \leq-1 & & \forall j \in D \\
g_{[K]}^{j}(v)=0 & & \forall j \in D, v \in V \backslash(F \cup\{j\}) \\
g_{[K]}^{j}(i) \leq h_{i}^{j} & & \forall j \in D, i \in F \\
h_{i}^{j} \leq y_{i} & & \forall j \in D, i \in F \\
f_{(u, v) ; k}^{j}+f_{(v, u) ; k}^{j} \leq x_{u v}^{k} & & \forall j \in D, k \in[K], u v \in E \\
\sum_{i \in S \cap F} h_{i}^{j}-\sum_{\bar{e} \in \delta(S)} z_{\bar{e}} \leq 0 & & \forall j \in D, S \subseteq V \backslash\{r\}: S \cap F \neq \emptyset \\
y_{r} & =1 & & \\
g_{[q, K]}^{j}(v) \leq 0 & & \forall j \in D, v \in V \backslash F, 1 \leq q \leq K \\
g_{[q, K]}^{j}(i)-\sum_{\bar{e} \in \delta(i)} z_{\bar{e}} \leq 0 & \\
x_{\bar{e}}^{k}, f_{e ; k}^{j}, y_{i}, z_{\bar{e}}, h_{i}^{j} \in\{0,1\} &
\end{array}
$$

Constraints (2) impose that at least one unit of flow leaves the clients. Constraints (3) are flow conservation constraints at non-facility nodes. Constraints (4) and (5) state that the flow only terminates at open facilities. Constraints (6) ensure that we install access links to support the flow. Finally, Constraints (7) state that if $i$ is the facility serving demand $j$ (the only $i$ for which $h_{i}^{j}=1$ ) then for each set $S$ containing $i$ and not containing the root there is a core link connecting $S$ with its complement. In other words, all open facilities are connected to the root via core links, where Constraint (8) defines the root facility. Constraints (9) and (10), called path monotonicity constraints, strengthen the linear relaxation of (IP-DDCFL). They ensure that the cable types along any path used to connect clients to facilities are nondecreasing from each client to its facility. We address the validity of these constraints below. Similar to that in [5], we can assume that the flow aggregated on an edge (in the optimum solution) never splits: once the flow from two clients share an edge they share the same set of edges on their paths to their facility. Consider any routing path connecting client $j$ to some facility $i$. As the flow never splits, the flow aggregated on edges of the path from $j$ to $i$ is nondecreasing. Therefore, as the access cable types obey economies of scale, we can conclude that the cable types along any routing path (in the optimal solution) are nondecreasing from each client to its facility.

The introduction of variables $h_{i}^{j}$ may seem artificial, however, in the Appendix we show that they are needed to achieve a constant integrality gap IP. 
We remark that the interesting variables of this IP formulation are $(f, x, y, z)=$ $\left(\left(f_{e ; k}^{j}\right),\left(x_{\bar{e}}^{k}\right),\left(y_{i}\right),\left(z_{\bar{e}}\right)\right)$. All the other quantities are written in terms of these variables.

\subsection{Proof of Theorem 1}

Let (LP-DDCFL) be the linear program relaxation of (IP-DDCFL) and $(f, x, y, z)$ be an optimal solution to (LP-DDCFL). It is not hard to show that (LP-DDCFL) can be solved in polynomial time using, for example, the ellipsoid method. We show how to round this LP solution to an integer one at constant factor loss.

\subsubsection{Rounding Algorithm.}

We extend the rounding approach of [17] for the single-source buy-at-bulk problem to devise a rounding algorithm for DDCFL. Our algorithm has four phases.

\section{Preprocessing Phase:}

Pruning: We prune the set of access cable types such that all cables are considerably different. Similar to [17], this can be done without increasing the cost of the optimal solution too much.

Theorem 3 Given $\epsilon_{1}, \epsilon_{2} \in(0,1)$, we can prune the set of access cables so that for any $i, \sigma_{i+1}>\sigma_{i} / \epsilon_{1}$ and $\delta_{i+1}<\epsilon_{2} \cdot \delta_{i}$ hold, increasing the installation and routing costs of the optimal fractional solution by a factor of at most $1 / \epsilon_{1}$ and $1 / \epsilon_{2}$, respectively.

For the sake of notation, let $[K]$ be the set of cables left and let $(f, x, y, z)$ be the new solution of (LP-DDCFL) after the pruning stage. For each client $j$ and positive radius $R$, define $B(j, R)=\left\{v \in V: c_{j v} \leq R\right\}$ to be the moat centered at $j$. We say that two moats $B_{1}=B\left(j_{1}, R_{1}\right)$ and $B_{2}=B\left(j_{2}, R_{2}\right)$ overlap if $c_{j_{1} j_{2}} \leq R_{1}+R_{2}$. Define also $L_{k}^{j}=\sum_{e \in \mathbf{E}} f_{e ; k}^{j} c_{\bar{e}}$ which represents the estimated distance that the flow of client $j$ travels on cables of type $k$. Note that $C_{k}^{\text {route }}=\delta_{k} \sum_{j \in D} d_{j} L_{k}^{j}$.

Flow path decomposition: Every client $j$ sends (at least) one unit of flow from itself to open facilities, specified by the $f_{e,[K]}^{j}$ variables. We decompose this fractional flow into a set of paths $P_{j}$, with path $p \in P_{j}$ starting from $j$ and ending at some facility. Let $\phi(p)$ denote the amount of flow of path $p$.

Filtering: For a predefined constant $\theta \in(0,1)$ and for all $j \in D$, choose a subset of paths $\bar{P}_{j} \subseteq P_{j}$ such that $\phi_{j}:=\sum_{p \in \bar{P}_{j}} \phi(p) \geq \theta$, by selecting paths in increasing order of their lengths until their total $\phi(p)$-value is at least $\theta$. For each $j \in D$, let $\beta_{j}$ be the length of the longest path in $\bar{P}_{j}$. Define a new solution $(\bar{f}, \bar{x}, \bar{y}, \bar{z})$ as follows. For each client $j \in D$, scale the amount of flow sent across each $P \in \bar{P}_{j}$ by $1 / \phi_{j}$ and set the flow sent across each $P \in P_{j}-\bar{P}_{j}$ to 0 . The new flow $\bar{f}$ (and hence $\bar{h}$ ) is derived naturally from this new path decomposition. For each cable $k \in[K]$ and edge $\bar{e} \in E$, define $\bar{x}_{\bar{e}}^{k}$ as $x_{\bar{e}}^{k} / \theta$ if there exists some $j$ with $\bar{f}_{e^{\prime} ; k}^{j}>0$, where $e^{\prime} \in \mathbf{E}$ is one of the two arcs associated to $\bar{e}$; and 0 otherwise. For each $i$, 
set $\bar{y}_{i}=\min \left\{y_{i} / \theta, 1\right\}$. And finally for each $\bar{e} \in E$, set $\bar{z}_{\bar{e}}=\min \left\{z_{\bar{e}} / \theta, 1\right\}$. It is easy to show that this solution is feasible for (LP-1).

Two important points: first, the solution $(\bar{f}, \bar{x}, \bar{y}, \bar{z})$ is such that the entire demand of client $j$ is satisfied by open facilities on the moat $B\left(j, \beta_{j}\right)$. The second property is the following bound which is useful for the analysis. Let $\widetilde{P}_{j} \subseteq P_{j}$ be the set of paths with lengths at least $\beta_{j}$. Then, $\widetilde{P}_{j}$ includes all paths in $P_{j} \backslash \bar{P}_{j}$ and at least one path, say $p^{*}$ (the longest) of $\bar{P}_{j}$. We conclude that $\sum_{p \in \widetilde{P}_{j}} \phi(p) \geq$ $\sum_{p \in P_{j}} \phi(p)-\sum_{p \in \bar{P}_{j} \backslash\left\{p^{*}\right\}} \phi(p) \geq 1-\theta$, and so

$$
\sum_{k=1}^{K} L_{k}^{j}=\sum_{p \in P_{j}} \sum_{e \in p} \phi(p) c_{\bar{e}} \geq \sum_{p \in \widetilde{P}_{j}} \phi(p) \sum_{e \in p} c_{\bar{e}} \geq \beta_{j}(1-\theta) .
$$

\section{Facility Selection Phase:}

Moat selection: For a predefined constant $\eta>1$, we consider the set of moats $\mathcal{B}_{\eta}=\left\{B\left(j, \eta \beta_{j}\right): j \in D\right\}$ around clients. We choose a maximal set $\mathcal{B}^{\prime} \subseteq \mathcal{B}_{\eta}$ of moats which do not overlap. We do this by processing the moats in $\mathcal{B}_{\eta}$ in increasing order of their radii, and greedily adding them to $\mathcal{B}^{\prime}$ so that for each pair of selected moats in $\mathcal{B}^{\prime}$ with centers $j, j^{\prime} \in D, B\left(j, \eta \beta_{j}\right)$ and $B\left(j^{\prime}, \eta \beta_{j^{\prime}}\right)$ do not overlap. Let $S_{\text {core }}$ be the set of clients with moats in $\mathcal{B}^{\prime}$. Observe that for the artificial root client $r^{*}$, we have $\beta_{r^{*}}=0$ and so $r^{*} \in S_{\text {core. }}$.

Facility opening: For each $j \in S_{\text {core, }}$ let $F_{j}=\left\{i: \bar{h}_{i}^{j}>0\right\}$ be the facilities fractionally serving demand from $j$ with respect to solution $(\bar{f}, \bar{x}, \bar{y}, \bar{z})$. By the first property noted at the end of the preprocessing phase, $F_{j} \subseteq B\left(j, \eta \beta_{j}\right)$, hence $\left\{F_{j}: j \in S_{\text {core }}\right\}$ consist of disjoint sets. On each $F_{j}$ we open the facility $i_{j}$ with lowest opening cost. In particular, the root $r$ is opened since $F_{r^{*}}=\{r\}$. Let $I$ be the set of facilities opened on this stage. The basic idea of this part of the algorithm is inspired by [15].

For the purpose of analysis, associate each client with a special facility denoted as its $(K+1)$-st proxy. Formally, for each $j \in S_{\text {core }}$ we set $\operatorname{proxy}_{K+1}(j)=i_{j}$. For the remaining clients $j \in D \backslash S_{\text {core }}$, we set $\operatorname{proxy}_{K+1}(j)=\operatorname{proxy}_{K+1}\left(j^{\prime}\right)$, where $j^{\prime} \in S_{\text {core }}$ is the center of the smallest moat in $\mathcal{B}^{\prime}$ that overlapped with $B\left(j, \eta \beta_{j}\right)$. Since the moats in $\mathcal{B}^{\prime}$ were added in increasing radii and (12), we get

$$
c\left(j, \operatorname{proxy}_{K+1}(j)\right) \leq(1+2 \eta) \beta_{j} \leq \frac{(1+2 \eta)}{(1-\theta)} \sum_{q=1}^{K} L_{q}^{j} \quad \forall j \in D .
$$

Core Network Phase: Consider the graph $G^{K+1}$ obtained from $G$ by contracting the nodes of each $F_{j}$ into single nodes, for $j \in S_{\text {core }}$. We construct an approximately optimal Steiner tree $T^{\prime}$ in $G^{K+1}$ having the contracted nodes as terminals. To do this, we find an approximate Steiner tree whose cost is within a factor 2 of the cut-based relaxation. The edges of $T^{\prime}$ form a forest in $G$ which touches a subset of the facilities in $F_{j}$, called $\tilde{F}_{j}$, which may not include the open facility $i_{j}$; see Figure 4(a). In order to connect all the open facilities together, we augment $T^{\prime}$ with the stars $Q_{j}=\left\{j i: i \in \tilde{F}_{j} \cup\left\{i_{j}\right\}\right\}, j \in S_{\text {core }}$; see Figure $4(\mathrm{~b})$. Let $T^{\text {core }}$ be the resulting tree, after possibly canceling some cycles. To conclude this stage, we install core cables on $T^{\text {core }}$. 


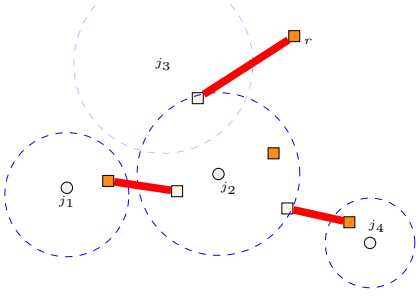

(a)

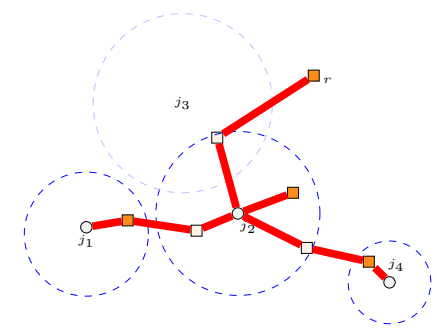

(b)

Fig. 4: An illustration of the core network installation phase on a simple instance where $S_{\text {core }}=\left\{r^{*}, j_{1}, j_{2}, j_{4}\right\}$.

Access Network Phase: We construct the access network in a top-down manner, installing cables progressively in stages numbered from $i=K$ to 1 . Let $T_{K+1}$ be a minimum spanning tree on the graph induced by the set $I$ of open facilities, and connect them using an artificial cable type $K+1$. This tree won't appear in the end, as it will be replaced by the core network. In stage $i$, we augment the current tree $T_{i+1}$, which uses only cables of type $i+1$ or higher, by installing cables of type $i$. Define $\bar{L}_{k}^{j}$ to be $\sum_{e \in \mathbf{E}} \bar{f}_{e ; k}^{j} \cdot c_{e}$. This estimates the distance that flow from $j$ goes on cable type $k$. Let $\bar{R}_{l}^{j}=\sum_{k=1}^{l-1} \bar{L}_{k}^{j}$ be the estimated distance beyond which the flow from $j$ uses cable type $l$ or higher in the new fractional solution. Intuitively, $\bar{R}_{l}^{j}$ tells us how far from $j$ to go before the LP solution installs access cable types $l$ or higher. Stage $i$ consists of two steps:

Step 1. Moat Selection: For predefined $\gamma>\zeta>1$, we construct the set of moats $\mathcal{B}_{\gamma}^{i}=\left\{B\left(j, \gamma \bar{R}_{i}^{j}\right): j \in D\right\}$ around all clients. We define $\hat{S}_{i}$ to be the set of clients whose moats intersect $T_{i+1}$. For each $j \in \hat{S}_{i}$ remove moat $B\left(j, \gamma \bar{R}_{i}^{j}\right)$ from $\mathcal{B}_{\gamma}^{i}$. Similar to what we did for the core network, we choose a maximal set $\mathcal{B}^{i} \subseteq \mathcal{B}_{\gamma}^{i}$ of moats which do not overlap by selecting moats from $\mathcal{B}_{\gamma}^{i}$ in increasing order of their radii. Let $S_{i}$ be the set of clients whose moats are selected in round $i$.

Step 2. Cable type $i$ installation: We construct the set $\mathcal{B}_{\zeta}^{i}=\left\{B\left(j, \zeta \bar{R}_{i}^{j}\right): j \in S_{i}\right\}$ of moats around clients in $S_{i}$. We obtain a graph $G^{i}$ from $G$ by contracting each moat in $\mathcal{B}_{\zeta}^{i}$ into a super-node, and the current tree $T_{i+1}$ into a super-node called $r_{i+1}$. We then construct an approximately optimal Steiner tree in $G^{i}$ (with integrality gap bound 2), where the terminals are all the super-nodes. By decontracting the supernodes, we get a forest in $G$ touching at least one node in $T_{i+1}$ and one node from each moat (see Figure $5(\mathrm{a})$ ). To get a tree, called $\tilde{T}_{i}$, from the resulting forest, we add direct edges from each client $j \in S_{i}$ to each node of $B\left(j, \zeta \bar{R}_{i}^{j}\right)$ that is incident on the forest and then we cancel cycles (see Figure 5(b)); we remark that this crucial step of adding direct edges is missing from the uSSBB-approximation in [17], even though it seems necessary for both that algorithm and ours to work.

Using Khuller et al.'s technique [12], we then convert tree $\tilde{T}_{i}$ rooted at $r_{i+1}$, into an $(\alpha, \beta)$-Light Approximate Shortest-path Tree (LAST), for parameters $\beta=\frac{\alpha+1}{\alpha-1}$ and $\alpha>1$ to be chosen later. Let $\mathrm{LAST}_{i}$ be the resulting tree. The LAST algorithm [12] transforms tree $\tilde{T}_{i}$ into $\mathrm{LAST}_{i}$ with $c\left(\mathrm{LAST}_{i}\right) \leq \beta c\left(\tilde{T}_{i}\right)$ such that the path 


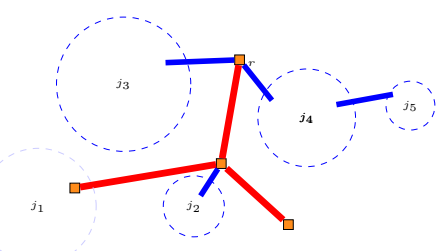

(a)

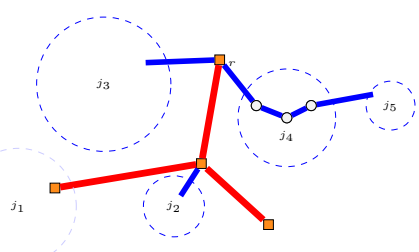

(b)

Fig. 5: An illustration of the access network phase where $i=K$.

length of any vertex $v$ to root $r_{i+1}$ in $\mathrm{LAST}_{i}$ is at most $\alpha$ times the length of a shortest $v-r_{i+1}$ path in $G^{i}$. We decontract the moats and install cables of type $i$ on the edges of $\mathrm{LAST}_{i}$. Let $T_{i}=T_{i+1} \cup \operatorname{LAST}_{i}$.

For the purpose of analysis, for each $j \in S_{i}$, we call an arbitrary node in its moat which is connected to $\operatorname{LAST}_{i}$ as the proxy, denoted by $\operatorname{proxy}_{i}(j)$. For the clients $j \in \hat{S}_{i}$, we define $\operatorname{proxy}_{i}(j)$ to be an arbitrary node in $B\left(j, \gamma \bar{R}_{i}^{j}\right) \cap T_{i+1}$. For the remaining clients $j^{\prime} \in D \backslash S_{i} \cup \hat{S}_{i}$, we define $\operatorname{proxy}_{i}\left(j^{\prime}\right)$ to be $\operatorname{proxy}_{i}(j)$, where $j \in S_{i}$ is the center of the smallest moat in $\mathcal{B}^{i}$ that overlapped with $B\left(j^{\prime}, \gamma \bar{R}_{i}^{j^{\prime}}\right)$. It is easy to verify that $c\left(j \operatorname{proxy}_{i}(j)\right) \leq 3 \gamma \bar{R}_{i}^{j} \leq \frac{3 \gamma}{\theta} \sum_{k=1}^{i-1} L_{k}^{j}$. If we set $\Delta=\max \left\{\frac{1+2 \eta}{1-\theta}, \frac{3 \gamma}{\theta}\right\}$, then by the previous inequality and (13), we get

$$
c\left(j, \operatorname{proxy}_{i+1}(j)\right) \leq \Delta \cdot \sum_{q=1}^{i} L_{q}^{j}, \quad \forall j \in D, 1 \leq i \leq K
$$

which will be useful in bounding the routing cost.

Finally, note that $R_{1}^{j}=0$ for all $j$. This means that in the first step of the last stage, $S_{1}$ consists of all clients that have not been connected to the current tree. Therefore, at the end of the last stage, $T_{1}$ is a tree spanning all clients and open facilities. The access network we return consists of the forest obtained by removing the artificial tree $T_{K+1}$ from $T_{1}$.

\subsubsection{Analysis.}

Let $C^{* \text { op }}, C^{* \text { core }}, C^{* \text { fixed }}$, and $C^{* \text { route }}$ be the opening cost, core installation cost, fixed installation cost, and routing cost paid by the LP optimum, respectively (see (1)). And let $C^{\text {op }}, C^{\text {core }}, C^{\text {fixed }}$, and $C^{\text {route }}$ be the ones paid by our algorithm. Let gap $_{\mathrm{ST}}$ denote the upper bound on the integrality gap of the cut based formulation of Steiner tree problem, which is 2. Let OPT be the cost of LP optimum. The following lemma bounds the opening cost.

Lemma 1 The opening cost of the returned solution is at most $\frac{1}{\theta} C^{* o p}$.

Proof The cost of facility $i_{j}$ can be bounded by using (2)-(5), and the fact that $i_{j}$ was chosen as the cheapest facility of $F_{j} \subseteq B\left(j, \beta_{j}\right)$, as follows

$$
\mu_{i_{j}}=\mu_{i_{j}} \cdot \frac{1}{\phi_{j}} \sum_{p \in \bar{P}_{j}} \phi(p)=\frac{1}{\phi_{j}} \sum_{i \in F_{j}} \mu_{i_{j}} h_{i}^{j} \leq \frac{1}{\theta} \sum_{i \in F_{j}} \mu_{i} y_{i} .
$$


As sets $F_{j}, j \in S_{\text {core }}$ are disjoint, the total opening cost is at most $\frac{1}{\theta} \cdot \sum_{i \in F} y_{i} \mu_{i}=$ $\frac{1}{\theta} C^{* \text { op }}$.

Lemma 2 The cost of core link installation is at most $\frac{\eta+1}{\theta(\eta-1)} \cdot g a p_{S T} \cdot C^{* \text { core }}$.

Proof By (7), one can verify that $\sum_{\bar{e} \in \delta^{+}(S)} \bar{z}_{\bar{e}} \geq 1$ holds for any arbitrary set $S \subset V$ that contains all facilities in $F_{j}$ (for some $j$ ) and it does not contain $r$. This means that $\bar{z}$ is a feasible fractional solution to the cut based LP relaxation of the Steiner tree problem on the graph $G^{K+1}$ (see the core network phase) whose terminals are all the contracted sets $F_{j}$ (recall that $F_{r^{*}}=\{r\}$ ). In particular, the Steiner tree $T^{\prime}$ found in the core network phase has cost at $\operatorname{most}_{\operatorname{gap}} \mathrm{ST}_{\overline{\mathrm{e}} \in E} c_{\bar{e}} \bar{z}_{\bar{e}}$. The cost of the extra edges included in the final tree $T^{\text {core }}$ (i.e., the union of all stars $Q_{j}$ ) can be charged to the cost of $T^{\prime}$ as follows.

For each facility $\tilde{i}_{j}$ in $\tilde{F}_{j}$ let $p\left(\tilde{i}_{j}\right)$ be any path in $T^{\prime}$ connecting $\tilde{i}_{j}$ (which is inside $\left.B\left(j, \beta_{j}\right)\right)$ to a node $v$ outside $B\left(j, \eta \beta_{j}\right)$, we note that the length of $p\left(\tilde{i}_{j}\right)$ is at least $(\eta-1) \beta_{j}$. By a similar argument, if $p\left(\tilde{i}_{j}\right)=p\left(\tilde{i}_{k}\right)$ where $\tilde{i}_{j} \in \tilde{F}_{j}$ and $\tilde{i}_{k} \in \tilde{F}_{k}$, then we can use the fact that $B\left(j, \eta \beta_{j}\right)$ and $B\left(k, \eta \beta_{k}\right)$ do not overlap to conclude that the length of the path connecting $\tilde{i}_{j}$ to $\tilde{i}_{k}$ in $T^{\prime}$ is at least $(\eta-1)\left(\beta_{j}+\beta_{k}\right)$. Therefore, the total cost of the union of all $Q_{j}$ is at most

$$
\sum_{j \in S_{\mathrm{core}}}\left(c\left(j i_{j}\right)+\sum_{\tilde{i}_{j} \in \tilde{F}_{j}} c\left(j \tilde{i}_{j}\right)\right) \leq 2 \sum_{j \in S_{\text {core }}} \sum_{\tilde{i}_{j} \in \tilde{F}_{j}} \beta_{j} \leq 2 \sum_{e \in T^{\prime}} \frac{c(e)}{\eta-1} .
$$

Summing up, the cost of $T^{\text {core }}$ is at most $1+\frac{2}{(\eta-1)}$ times the cost of $T^{\prime}$, and therefore it is at most $\frac{\eta+1}{\theta(\eta-1)} \cdot \operatorname{gap}_{\mathrm{ST}} \cdot \sum_{e \in E} c_{\bar{e}} z_{\bar{e}}$.

In the following, extending the ideas from [17] for uSSBB, we bound the fixed cost and routing cost of the cables installed at stage $i$ of the access network phase of our algorithm, denoted by $C_{i}^{\text {fixed }}$ and $C_{i}^{\text {route }}$, respectively.

Lemma $3 C_{i}^{\text {fixed }} \leq \sigma_{i} \cdot \operatorname{gap}_{S T} \cdot \frac{\gamma \beta \zeta}{(\gamma-\zeta)(\zeta-1) \theta}\left(\sum_{q=i}^{K} \frac{1}{\sigma_{q}} C_{q}^{* \text { fixed }}+\frac{1}{M} C^{* \text { core }}\right)$.

Proof Let $S$ be an arbitrary subset of $V \backslash\{r\}$ that contains $B\left(j, \zeta \bar{R}_{i}^{j}\right)$ and let $\bar{b}_{q ; S}^{j}:=\sum_{e \in \delta^{+}(S)} \bar{f}_{e, q}^{j}$ denote the amount of flow from $j$ that crosses the boundary of $S$ via cables of type $q$. We note that the flow we are considering may travel from $j$ to the boundary of $S$ using any cables of type $q$ or less than $q$ (due to the path monotonicity constraints) but it must use cables of type $q$ while crossing the boundary of $S$.

In the following we first show that $\sum_{q=1}^{i-1} \bar{b}_{q ; S}^{j} \leq \frac{1}{\zeta}$. Consider some $q<i$. As $\bar{b}_{q ; S}^{j}$ travels a distance of at least $\zeta \bar{R}_{i}^{j}$ (on cables of type $q$ or less than $q$ ), it contributes at least $\bar{b}_{q ; S}^{j} \cdot \zeta \bar{R}_{i}^{j}$ units to $\bar{R}_{i}^{j}=\sum_{k=1}^{i-1} \bar{L}_{k}^{j}$. As the contributions from each $q$ are disjoint, we have $\bar{R}_{i}^{j} \geq \sum_{q=1}^{i-1} \bar{b}_{q ; S}^{j} \zeta \bar{R}_{i}^{j}$, which implies that $\sum_{q=1}^{i-1} \bar{b}_{q ; S}^{j} \leq \frac{1}{\zeta}$. This together with the LP constraints guarantee that $\sum_{q=i}^{K} \bar{b}_{q ; S}^{j}+\sum_{e \in \delta^{+}(S)} \bar{z}_{e} \geq$ $1-\frac{1}{\zeta}$ and hence $\sum_{e \in \delta^{+}(S)}\left(\sum_{q=i}^{K} \bar{x}_{e}^{q}+\bar{z}_{e}\right) \geq 1-\frac{1}{\zeta}$ by the definition of $\bar{b}_{q ; S}^{j}$ and constraints (6). This means that the vector $\bar{z}+\sum_{q=i}^{K} \bar{x}^{q}$, scaled by a factor $\frac{\zeta}{\zeta-1}$, is a feasible fractional solution to the LP relaxation of the Steiner tree connecting 
balls $B\left(j, \zeta \bar{R}_{i}^{j}\right)$ to $T_{i+1}$. Therefore, the cost of the Steiner tree computed in step 2 of the access network phase can be bounded by

$$
\frac{\operatorname{gap}_{\mathrm{ST}} \zeta}{\zeta-1}\left(\sum_{e \in E} \sum_{q=i}^{K} c_{e} \bar{x}_{e}^{q}+\sum_{e \in E} c_{e} \bar{z}_{e}\right) \leq \frac{\operatorname{gap}_{\mathrm{ST}} \zeta}{(\zeta-1) \theta}\left(\sum_{q=i}^{K} \frac{1}{\sigma_{q}} C_{q}^{* \text { fixed }}+\frac{1}{M} C^{* \text { core }}\right)
$$

Similar to Lemma 2, one can show that the cost of extra edges of $\tilde{T}_{i}$, added after un-contracting the moats, is at most $\frac{\zeta}{\gamma-\zeta}$ times the cost of the current forest. Altogether, the cost of the $\mathrm{LAST}_{i}$ tree is at most

$$
\begin{aligned}
c\left(\mathrm{LAST}_{i}\right) & \leq \beta\left(1+\frac{\zeta}{\gamma-\zeta}\right) \cdot \frac{\operatorname{gap}_{\mathrm{ST}} \zeta}{(\zeta-1) \theta}\left(\sum_{q=i}^{K} \frac{1}{\sigma_{q}} C_{q}^{* \text { fixed }}+\frac{1}{M} C^{* \text { core }}\right) \\
& =\operatorname{gap}_{\mathrm{ST}} \frac{\beta \gamma \zeta}{(\gamma-\zeta)(\zeta-1) \theta}\left(\sum_{q=i}^{K} \frac{1}{\sigma_{q}} C_{q}^{* \text { fixed }}+\frac{1}{M} C^{* \text { core }}\right)
\end{aligned}
$$

Lemma $4 C_{i}^{\text {route }} \leq \Delta \delta_{i} \alpha \sum_{q=1}^{i}(1+\alpha)^{i-q} \frac{1}{\delta_{q}} C_{q}^{* \text { route }}$

Proof Let $T=\bigcup_{i=1}^{K} \operatorname{LAST}_{i}$ be the access network (forest) constructed by our algorithm and let $V_{i}$ be the set of nodes via which flow routing from clients toward facilities enters the $\mathrm{LAST}_{i}$ tree; we assume $V_{1}=D$. Also, let $d_{T}(u, v)$ be the distance between $u$ and $v$ on $T$.

The proof of the lemma is by induction on $i$. Since we install cables of type 1 on the $\mathrm{LAST}_{1}$ tree, and also $\operatorname{proxy}_{2}(j)$ lies in $T_{2}$, we have $d_{T}\left(j, T_{2}\right) \leq \alpha c\left(j, \operatorname{proxy}_{2}(j)\right)$. Hence by using (14), we get

$$
C_{1}^{\text {route }}=\delta_{1} \sum_{j \in D} d_{j} \cdot d_{T}\left(j, T_{2}\right) \leq \Delta \delta_{1} \alpha \sum_{j \in D} d_{j} L_{1}^{j} \leq \Delta \delta_{1} \alpha \frac{1}{\delta_{1}} C_{1}^{* \text { route }}
$$

which concludes the case $i=1$. Assume now, that the claim holds for all $l<i$. Then the total cost of routing along cables of type $i$ can be bounded as the following.

$$
C_{i}^{\text {route }}=\delta_{i} \sum_{v \in V_{i}} D_{v} \cdot d_{T}\left(v, T_{i+1}\right) \leq \delta_{i} \alpha\left[\sum_{p=1}^{i-1} \frac{C_{p}^{\text {route }}}{\delta_{p}}+\sum_{j \in D} d_{j} \Delta \sum_{q=1}^{i} L_{q}^{j}\right]
$$

where $D_{v}$ is the amount of demand routed through $\mathrm{LAST}_{i}$ via node $v$. The above inequality holds because the cost of of routing the demand from $v$ to $T_{i+1}$ on $T$ can be bounded by the cost of routing the demand back (via forest defined by the edges of $T$ that have cables of types $1, \ldots, i-1$ ) to its source (sources), say $j$, and then from there (directly) to $\operatorname{proxy}_{i+1}(j)$, which lies in $T_{i+1}$, using the triangle inequality and (14). Note that this is true because we install cables on the $\mathrm{LAST}_{i}$ trees. Recall $C_{q}^{\text {route }}=\delta_{q} \sum_{j \in D} d_{j} L_{q}^{j}$. Therefore by (15) and induction hypothesis 
we get

$$
\begin{aligned}
C_{i}^{\text {route }} & \leq \Delta \delta_{i} \alpha\left[\sum_{p=1}^{i-1} \alpha \sum_{q=1}^{p}(1+\alpha)^{p-q} \frac{C_{q}^{* \text { route }}}{\delta_{q}}+\sum_{q=1}^{i} \frac{C_{q}^{* \text { route }}}{\delta_{q}}\right] \\
& =\Delta \delta_{i} \alpha\left[\sum_{q=1}^{i-1} \frac{C_{q}^{* \text { route }}}{\delta_{q}}\left[\alpha \sum_{p=q}^{i-1}(1+\alpha)^{p-q}\right]+\sum_{q=1}^{i-1} \frac{C_{q}^{* \text { route }}}{\delta_{q}}+\frac{C_{i}^{* \text { route }}}{\delta_{i}}\right] \\
& =\Delta \delta_{i} \alpha\left[\sum_{q=1}^{i-1} \frac{C_{q}^{* \text { route }}}{\delta_{q}}(1+\alpha)^{i-q}+\frac{C_{i}^{* \text { route }}}{\delta_{i}}\right]=\Delta \delta_{i} \alpha \sum_{q=1}^{i} \frac{C_{q}^{* \text { route }}}{\delta_{q}}(1+\alpha)^{i-q} .
\end{aligned}
$$

By Lemma 3, Theorem 3, and by summing over all cable types, the fixed cost paid by the algorithm can be bounded as follows.

$$
\begin{aligned}
C^{\text {fixed }} & \leq \operatorname{gap}_{\mathrm{ST}} \cdot \frac{\gamma \beta \zeta}{(\gamma-\zeta)(\zeta-1) \theta}\left[\sum_{s=1}^{K} C_{s}^{* \text { fixed }}\left(\sum_{i \leq s} \frac{\sigma_{i}}{\sigma_{s}}\right)+C^{* \operatorname{core}}\left(\sum_{i=1}^{K} \frac{\sigma_{i}}{M}\right)\right] \\
& \leq \operatorname{gap}_{\mathrm{ST}} \cdot \frac{\gamma \beta \zeta}{(\gamma-\zeta)(\zeta-1) \theta\left(1-\epsilon_{1}\right)}\left[C^{* \text { fixed }}+C^{* \text { core }}\right] .
\end{aligned}
$$

Similarly, by using Lemma (4), we bound the routing cost as follows.

$$
\begin{aligned}
C^{\text {route }} & \leq \Delta \alpha \sum_{i=1}^{K} \sum_{s=1}^{i}(1+\alpha)^{i-s} \frac{\delta_{i}}{\delta_{s}} C_{s}^{* \text { route }} \leq \Delta \alpha \sum_{i=1}^{K} \sum_{s=1}^{i}\left((1+\alpha) \cdot \epsilon_{2}\right)^{i-s} C_{s}^{* \text { route }} \\
& \leq \Delta \alpha \sum_{s=1}^{K} C_{s}^{* \text { route }} \sum_{i \geq s}\left((1+\alpha) \cdot \epsilon_{2}\right)^{i-s} \leq \frac{\Delta \alpha}{1-\epsilon_{2}(1+\alpha)} \cdot C^{\text {route }} .
\end{aligned}
$$

Using (16), (17), Lemmas 1 and 2, the total cost of our solution is at most $\frac{1}{\theta} C^{* \text { op }}+\frac{(\eta+1) \operatorname{gap}_{\mathrm{ST}}}{\theta(\eta-1)} C^{* \text { core }}+\frac{\gamma \beta \zeta \cdot \operatorname{gap}_{\mathrm{ST}}\left(C^{* \text { fixed }}+C^{* \text { core }}\right)}{(\gamma-\zeta)(\zeta-1) \theta\left(1-\epsilon_{1}\right)}+\frac{\Delta \alpha}{1-\epsilon_{2}(1+\alpha)} C^{* \text { route }}$.

Finally, using Theorem 3, we can bound the cost of our solution by $\leq \max \left(\frac{1}{\epsilon_{1}} \cdot \frac{\gamma \beta \zeta \cdot \operatorname{gap}_{\mathrm{ST}}}{(\gamma-\zeta)(\zeta-1) \theta\left(1-\epsilon_{1}\right)}+\frac{(\eta+1) \operatorname{gap}_{\mathrm{ST}}}{\theta(\eta-1)}, \frac{1}{\epsilon_{2}} \cdot \frac{\Delta \alpha}{1-\epsilon_{2}(1+\alpha)}\right)$ OPT.

This completes the proof of Theorem 1. Setting $\alpha=1.47, \gamma=4.10, \epsilon_{1}=0.50$, $\epsilon_{2}=0.20, \theta=0.78, \eta=1.27$ and $\zeta=2$ and recalling gap ST $_{\mathrm{ST}}=2$, inequality (18) implies that the integrality gap of (IP-DDCFL) is no more than 234. Thus, we obtain the first LP based (deterministic) algorithm for DDCFL and thereby for BBCFL.

\section{Buy-at-Bulk Facility Location}

In this section we study the integrality gap of an LP formulation for the buy-atbulk facility location problem. As with BBCFL, we consider the deep-discount variant of BBFL, namely DDFL. Note that similar to the relation between BBCFL and DDCFL, one can transform between BBFL and DDFL with a factor 2 loss. 


\subsection{IP Formulation}

Similar to Section 2.1, DDFL can be formulated as follows:

$$
\begin{array}{cl}
\min C^{\text {op }}+C^{\text {fixed }}+C^{\text {route }} & \\
(2),(3),(6),(9) & \\
g_{[K]}^{j}(i) \leq y_{i} & \forall j \in D, i \in F \\
g_{[q, K]}^{j}(i)-y_{i} \leq 0 & \forall j \in D, i \in F, 1 \leq q \leq K \\
x_{\bar{e}}^{k}, f_{e ; k}^{j}, y_{i} \in\{0,1\} &
\end{array}
$$

We do not need the $z$ and $h_{i}^{j}$ variables anymore, as they were used to model the connectivity requirements among facilities. Constraints (19) state that the flow only ends at open facilities, and constraints (9) and (20) force the path monotonicity discussed in Section 2.1.

\subsection{Proof of Theorem 2}

Let $(f, x, y)$ be the optimal solution to the LP relaxation of (IP-DDFL). We shall show that this fractional solution can be rounded to an integer solution increasing the total cost by a factor of $O(K)$.

\subsubsection{Rounding Algorithm.}

Our rounding algorithm will follow the same general ideas of that for DDCFL, but we replace the core network and access network phases by a single one denoted network phase. Another key difference is that we may open facilities at any stage of the network phase. Ultimately, this is why our integrality gap bound is $O(K)$ as we have to overestimate and bound the opening cost in each of the $K$ stages by the total opening cost paid by the LP.

Preprocessing Phase: Apply the preprocessing phase (pruning, flow path decomposition and filtering) of Section 2.2.1, disregarding variables $z$. Let $(\bar{f}, \bar{x}, \bar{y})$ be the solution after this phase.

Initial Facility Selection Phase. Perform the facility selection phase of Section 2.2.1 but fixing $\eta=1$. Let $I^{\prime}$ be the set of facilities opened in this phase.

Network Phase: We construct a solution in a top-down manner, installing cables and possibly opening more facilities in stages, which we number from $i=K$ to 1 . We start with solution $\left(I_{K+1}, T_{K+1}\right)=\left(I^{\prime}, \emptyset\right)$. At stage $i$ we augment the current solution by (1) opening some extra facilities and (2) installing cables of type $i$. We do this while keeping the invariant that $T_{i}$ is a forest in $G$ such that each connected component contains an open facility of $I_{i}$.

Stage $i$ of this phase of our algorithm is similar to the $i$-th stage of the access network phase in Section 2.2.1 and works as follows: 
1. For a predefined constant $\gamma>\zeta>1$, construct the set of moats $B\left(j, \gamma \bar{R}_{j}^{i}\right)$ around clients $j \in D$. Remove the moats which intersect $T_{i+1}$ and select from the rest a maximal subset $\mathcal{B}^{i}$ of non-overlapping moats in increasing order of their radii. Let $S_{i}$ be the set of selected clients associated to $\mathcal{B}^{i}$ and construct the set $\mathcal{B}_{\zeta}^{i}=\left\{B\left(j, \zeta \bar{R}_{i}^{j}\right): j \in S_{i}\right\}$ of moats around clients in $S_{i}$.

2. Add a dummy node $\tilde{r}$ and connect it to every facility $v$ fractionally opened by the LP (with $\bar{y}_{v}>0$ ). Set the cost of each dummy edge $\tilde{e}=\tilde{r} v$ to be zero if facility $v \in I_{i+1}$; otherwise set it to be $f_{v}$. To simplify the analysis, associate each edge $\tilde{e}=\tilde{r} v$ with a variable $\tilde{x}_{\tilde{e}}$ equal to $\bar{y}_{v}$.

3. Contract each moat in $\mathcal{B}_{\zeta}^{i}$, and each component of $T_{i+1}$ into super-nodes. Call the contracted graph $\widetilde{G}$.

4. Construct an approximately optimal Steiner tree $\hat{T}$ on $\widetilde{G}$, where the terminals are $\tilde{r}$ and all the super-nodes. Without loss of generality we assume that $\hat{T}$ includes a dummy edge of cost 0 from $\tilde{r}$ to every super-node associated to a component of $T_{i+1}$ (or, more precisely, to each facility $v \in I_{i+1}$ ).

5. For each $v \in F \backslash I_{i+1}$, if edge $\tilde{r} v$ is in $\hat{T}$ then open facility $v$ and put it in $I_{i}$.

6. Set $I_{i}=I_{i} \cup I_{i+1}$.

7. Contract all the dummy edges that are contained in $\hat{T}$, and decontract the super-nodes associated to the moats. The edges from $\hat{T}$ form a forest in the resulting graph. To get a tree, add for each moat direct edges from its center to all nodes in the moat that are incident to $\hat{T}$. Let $\widetilde{T}$ be the resulting tree.

8. Using the LAST algorithm for appropriate parameters, transform $\widetilde{T}$ rooted at the contracted node containing $\tilde{r}$ into a tree called $\mathrm{LAST}_{i}$.

9. Install cables of type $i$ along $\operatorname{LAST}_{i}$ and let $T_{i}=T_{i+1} \cup \operatorname{LAST}_{i}$.

\subsubsection{Analysis.}

Let $C^{* \text { op }}, C^{* \text { fixed }}$, and $C^{* \text { route }}$ be the opening cost, fixed installation cost and routing cost paid by the optimum to the LP relaxation of (IP-DDFL), respectively.

Similar to Lemma 1 , the cost of facilities opened in the facility selection phase can be bounded as follows.

Lemma 5 The cost of facilities opened in the facility selection phase is $O(1) \cdot C^{* o p}$.

In the next lemma, we bound the fixed installation and opening cost incurred in the network phase of the algorithm.

Lemma 6 The total fixed cost and facility cost of the network phase is $O(1)$. $C^{* \text { fixed }}+O(K) \cdot C^{* o p}$.

Proof Consider Stage $i$ of this phase. Let $S$ be an arbitrary subset of $V \backslash\{r\}$ that contains $B\left(j, \zeta \bar{R}_{i}^{j}\right)$. Similar to Lemma 3, one can show that $\sum_{q=1}^{i-1} \bar{b}_{q ; S}^{j} \leq \frac{1}{\zeta}$, where $\bar{b}_{q ; S}^{j}:=\sum_{e \in \delta^{+}(S)} \bar{f}_{e, q}^{j}$ indicates the amount of flow from $j$ crossing the boundary of $S$ thorough cables of type $q$. This together with the LP constraints guarantee that $\sum_{q=i}^{K} \bar{b}_{q ; S}^{j}+\sum_{v \in S \cap F} \bar{y}_{v} \geq 1-\frac{1}{\zeta}$ and hence $\sum_{e \in \delta^{+}(S)}\left(\sum_{q=i}^{K} \bar{x}_{e}^{q}+\tilde{x}_{e}\right) \geq 1-\frac{1}{\zeta}$. This means that the vector $\tilde{x}+\sum_{q=i}^{K} \bar{x}^{q}$, scaled by a factor $\frac{\zeta}{\zeta-1}$, can be viewed as a feasible fractional solution to the LP relaxation the Steiner tree problem on $\widetilde{G}$ connecting balls $B\left(j, \zeta \bar{R}_{i}^{j}\right)$ to $T_{i+1}$. Therefore, the cost of this Steiner tree 
(including edge cost of Step 4 and opening cost of Step 5) can be bounded by

$$
\frac{\operatorname{gap}_{\mathrm{ST}} \zeta}{\zeta-1}\left(\sum_{e \in E} \sum_{q=i}^{K} c_{e} \bar{x}_{e}^{q}+\sum_{v \in F} \mu_{v} \tilde{x}_{\tilde{r} v}\right) \leq \frac{\operatorname{gap}_{\mathrm{ST}} \zeta}{(\zeta-1) \theta}\left(\sum_{q=i}^{K} \frac{1}{\sigma_{q}} C_{q}^{* \text { fixed }}+C^{* \mathrm{op}}\right)
$$

Similar to Lemma 3, the cost of the $\mathrm{LAST}_{i}$ tree can be bounded by

$$
\operatorname{gap}_{\mathrm{ST}} \cdot \frac{\gamma}{\gamma-\zeta} \cdot \frac{\beta \zeta}{(\zeta-1) \theta}\left(\sum_{q=i}^{K} \frac{1}{\sigma_{q}} C_{q}^{* \mathrm{fixed}}+C^{* \mathrm{op}}\right)
$$

Summing over all stages $1 \leq i \leq K$, we see the total fixed cost and facility cost of this phase is at most

$$
\operatorname{gap}_{\mathrm{ST}} \cdot \frac{\gamma}{\gamma-\zeta} \cdot \frac{\beta \zeta}{(\zeta-1) \theta} \sum_{i=1}^{K}\left(\sum_{q=i}^{K} \frac{\sigma_{i}}{\sigma_{q}} C_{q}^{* \text { fixed }}+C^{* \mathrm{op}}\right)
$$

Which is $O(1) \cdot C^{* \text { fixed }}+O(K) \cdot C^{* \text { op }}$ by Theorem 3 .

Similar to Lemma 4, we can bound the routing cost of each stage, and then the routing cost of the entire solution.

Lemma 7 The total routing cost of the solution is $O(1) \cdot C^{* \text { route }}$.

Using Lemmas 5, 6, and 7, the total cost of the solution is at most

$$
O(1)\left(C^{* \text { route }}+C^{* \text { fixed }}\right)+O(K) \cdot C^{* \text { op }}
$$

This completes the proof of Theorem 2 .

\section{Conclusions}

We have shown that the LP rounding framework for uSSBB given by [17] extends to facility location buy-at-bulk problems. Our integrality gap analysis roughly matches the known approximation ratios of combinatorial algorithms for BBCFL and BBFL, so the obvious open problem is to improve this analysis to derive better approximation algorithms. In particular, can we get an $O(1)$-approximation for BBFL? We were able to bound the gap by $O(1)$ for BBCFL by exploiting the fact that the facility core network is fractionally connected by the LP. However, in BBFL we do not have this property so we have to pay for the facility opening costs with a copy of the $y$-values in each stage.

A potentially easier problem is to get an $\alpha$-approximation for BBFL with running time $n^{f(k)}$ for some function $f$ where $\alpha$ is a constant that does not depend on $k$.

Acknowledgements A special thank to Babak Behsaz for helpful discussions. 


\section{References}

1. Bley, A., Rezapour, M.: Combinatorial approximation algorithms for buy-at-bulk connected facility location problems. Discrete Applied Mathematics 213, 34-46 (2016)

2. Chekuri, C., Khanna, S., Naor, J.S.: A deterministic algorithm for the cost-distance problem. Proceedings of the twelfth annual ACM-SIAM Symposium On Discrete Algorithms (SODA) pp. 232-233 (2001)

3. Eisenbrand, F., Grandoni, F., Rothvoß, T., Schäfer, G.: Connected facility location via random facility sampling and core detouring. Journal of Computer and System Sciences 76(8), 709-726 (2010)

4. Friggstad, Z., Rezapour, M., Salavatipour, M.R., Soto, J.A.: LP-based approximation algorithms for facility location in buy-at-bulk network design. Proceedings of the 14th International Symposium on Algorithms and Data Structures (WADS). pp. 373-385 (2015)

5. Garg, N., Khandekar, R., Konjevod, G., Ravi, R., Salman, F.S., Sinha, A.: On the integrality gap of a natural formulation of the single-sink buy-at-bulk network design problem. Proceedings of Integer Programming and Combinatorial Optimization (IPCO) pp. 170-184 (2001)

6. Grandoni, F., Rothvoß, T.: Network design via core detouring for problems without a core. Proceedings of International Colloquium on Automata, Languages, and Programming (ICALP) pp. 490-502 (2010)

7. Grandoni, F., Rothvoß, T.: Approximation algorithms for single and multi-commodity connected facility location. Proceedings of Integer Programming and Combinatorial Optimization (IPCO) pp. 248-260 (2011)

8. Grandoni, F., Rothvoß, T., Sanità, L.: From uncertainty to nonlinearity: Solving virtual private network via single-sink buy-at-bulk. Mathematics of Operations Research 36(2), 185-204 (2011)

9. Guha, S., Meyerson, A., Munagala, K.: A constant factor approximation for the single sink edge installation problem. SIAM Journal on Computing 38(6), 2426-2442 (2009)

10. Gupta, A., Kleinberg, J., Kumar, A., Rastogi, R., Yener, B.: Provisioning a virtual private network: a network design problem for multicommodity flow. Proceedings of the thirtythird annual ACM symposium on Theory of computing (STOC) pp. 389-398 (2001)

11. Jothi, R., Raghavachari, B.: Improved approximation algorithms for the single-sink buy-atbulk network design problems. Proceedings of Scandinavian Symposium and Workshops on Algorithm Theory (SWAT) pp. 336-348 (2004)

12. Khuller, S., Raghavachari, B., Young, N.: Balancing minimum spanning trees and shortestpath trees. Algorithmica 14(4), 305-321 (1995)

13. Meyerson, A., Munagala, K., Plotkin, S.: Cost-distance: Two metric network design. Proceedings of the 41st Annual Symposium on Foundations of Computer Science (FOCS). pp. 624-630 (2000)

14. Ravi, R., Sinha, A.: Approximation algorithms for problems combining facility location and network design. Operations Research 54(1), 73-81 (2006)

15. Shmoys, D.B., Tardos, É., Aardal, K.: Approximation algorithms for facility location problems. Proceedings of the annual ACM symposium on Theory of computing (STOC) pp. 265-274 (1997)

16. Swamy, C., Kumar, A.: Primal-dual algorithms for connected facility location problems. Algorithmica 40(4), 245-269 (2004)

17. Talwar, K.: The single-sink buy-at-bulk lp has constant integrality gap. Proceedings of Integer Programming and Combinatorial Optimization (IPCO) pp. 475-486 (2002)

\section{Appendix. A naive Model for DDCFL}

In this section, we show that an alternative, but perhaps more natural IP formulation for DDCFL has unbounded integrality gap. Consider the following integer programming formu- 
lation:

$$
\begin{aligned}
& \min C^{\text {op }}+C^{\text {core }}+C^{\text {fixed }}+C^{\text {route }} \\
& g_{[K]}^{j}(j) \leq-1 \quad \forall j \in D \\
& g_{[K]}^{j}(v)=0 \quad \forall j \in D, v \in V \backslash(F \cup\{j\}) \\
& f_{(u, v) ; k}^{j}+f_{(v, u) ; k}^{j} \leq x_{u v}^{k} \quad \forall j \in D, k \in[K], u v \in E \\
& y_{r}=1 \\
& g_{[q, K]}^{j}(v) \leq 0 \\
& g_{[q, K]}^{j}(i)-\sum_{\bar{e} \in \delta(i)} z_{\bar{e}} \leq 0 \\
& g_{[K]}^{j}(i) \leq y_{i} \\
& y_{i}-\sum_{\bar{e} \in \delta(S)} z_{\bar{e}} \leq 0 \\
& x_{\bar{e}}^{k}, f_{e ; k}^{j}, y_{i}, z_{\bar{e}} \in\{0,1\}
\end{aligned}
$$

The difference between this formulation and (IP-DDCFL) is that (IP-DDCFL-2) does not have variables $h$. We replace constraints (4) and (7) by (22) and (23) respectively.

Theorem 4 The integrality gap of (IP-DDCFL-2) can be arbitrarily large.

Proof Proof. Consider the instance described in Figure 6, where the square nodes represent facilities and the circle nodes represent clients. In this instance, $K=1$, i.e. we have a unique access cable type, and we set $\sigma=\delta=1$. The core cable has a cost (per unit length) equal to $M$ with $1<M<q$. For every facility $i \in\{1, \cdots p\}$, we set an opening cost of $\mu_{i}=1$. We also set $\mu_{n}=\infty$. The root facility $r$, which must be opened, has an opening cost of 0 . The distances are given by the metric completion of the edge costs depicted in the figure, where $L>>1$ is a constant larger than any other finite parameter of the instance.

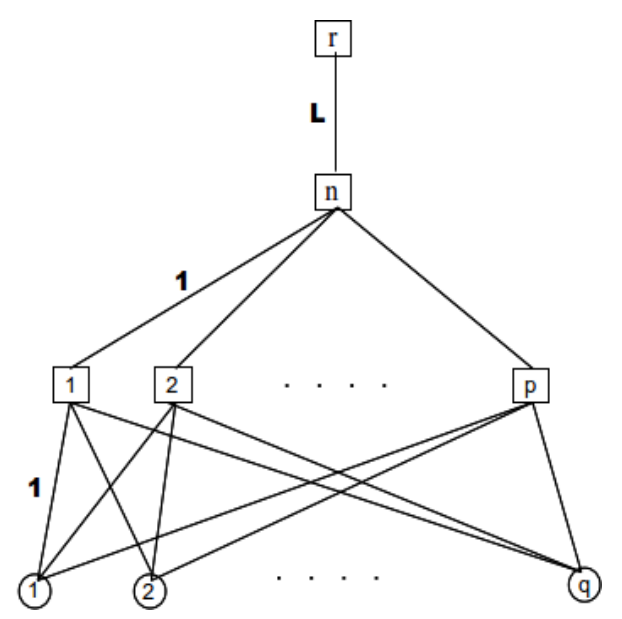

Fig. 6: An instance of DDCFL with $q$ clients of unit demands and $(p+2)$ potential facilities with facility $r$ as the root node. 
The optimal integral solution to this instance can connect all the clients to a fixed facility $i^{*} \in\{1, \cdots p\}$ via access links; note that facilities $\{1, \cdots p\}$ are (almost) collocated. Then this open facility is connected to the root node via (unopened) facility $n$ using core links.

However, the LP relaxation of (IP-DDCFL-2) can cheat and open all facilities $i \in\{1, \cdots p\}$ to the extends of $1 / p$ to serve clients demands. Then it can install core links to the extends of $1 / p$ on the edges connecting them (via node $n$ ) to the root node. This means that LP only pays $M \cdot L / p$ for the core link along edge $n r$, while the integral solution pays a cost of $M \cdot L$ on that for the same edge. Since $L$ was chosen as an arbitrarily large constant, this is the only relevant value to compare. Hence, the integrality gap is proportional to $p$ and thus it can be made arbitrarily large. 